\title{
Research on MATLAB-based Image Preprocessing
}

\author{
Zhengxi Hu ${ }^{a}$, Honghai Jiang ${ }^{b}$ and Tianyi Zhang ${ }^{c}$ \\ School of Kunming University of Science and Technology, Kunming 650000, China \\ a372396292@qq.com, b36078614@qq.com, '263628972@qq.com
}

Keywords: MATLAB; digital image processing; image pre-processing; de-noising; fuzzy image processing.

\begin{abstract}
On the basis of digital image processing, image preprocessing, including de-noising and fuzzy image processing, is carried out in the MATLAB software environment, and detailed processing steps and associated procedures are given. The results show that MATLAB plays an effective role in the image preprocessing.
\end{abstract}

\section{Introduction}

With the innovation of electronic technology and the development of various image processing software, such as, VC ++, Halcon, PS, etc., image preprocessing is widely used in various image processing. In this paper, the mean of field, median filtering, Gaussian filtering, and Lucy-Richardson algorithm are used in image processing and detailed steps for simulation process in the MATLAB are given which can be applied in a variety of image processing electronic systems.

\section{The image noise and de-noising}

Image noise. Factors in image which prevent people accepting the information can be called image noise. Common noises that are Gaussian noise and salt and pepper noise are to be analyzed in this paper.

Gaussian noise is a random noise; optionally take any Gaussian noise of $\mathrm{n}$ in instantaneous choice and the value is distributed according to the laws of Gaussian probability of n variables. Firstly, the Gaussian noise is entirely determined by its average and instantaneous covariance function. If the noise is stationary, the average value is independent of time, and covariance function becomes correlation function related to the difference of two instantaneities, which is equivalent to the power spectral density in the sense. Secondly, Gaussian noise may be generated by a large number of independent pulses, so that in any finite time, each pulse value in all of the pulse and the sum of these pulses can be negligible. Thirdly, the thermal noise, shot noise and quantum noise are actually Gaussian noise.

Salt and pepper noise is black and white bright scotoma noise generated by image sensor, transmission channel and decoding processing, etc., which is often caused by the image cut. Salt and pepper noise refers to two types of noise, namely, salt noise and pepper noise, in which salt is white and pepper is black. The former is a high gray noise, and the latter belongs to the low gray noise. Generally, these two kinds of noise occur simultaneously, which are presented in black and white spots in the image.

De-noising. Neighborhood average method. Mathematical expression of neighborhood average method for smooth image processing is as follows:

$$
g(x, y)=\frac{1}{M} \sum_{(m, n) \in S} f(x-m, y-n) \cdot
$$

In the expression, $\mathrm{M}$ is the total number of pixels contained in the neighborhood field of $\mathrm{S}$; $\mathrm{S}$ is the determinate neighborhood field which does not include the $\mathrm{x}$ and $\mathrm{y}$ point. The original image of salt and pepper noise and Gaussian noise, and the results of image processing are shown as Fig. 1. MATLAB code is shown in Fig. 2. 


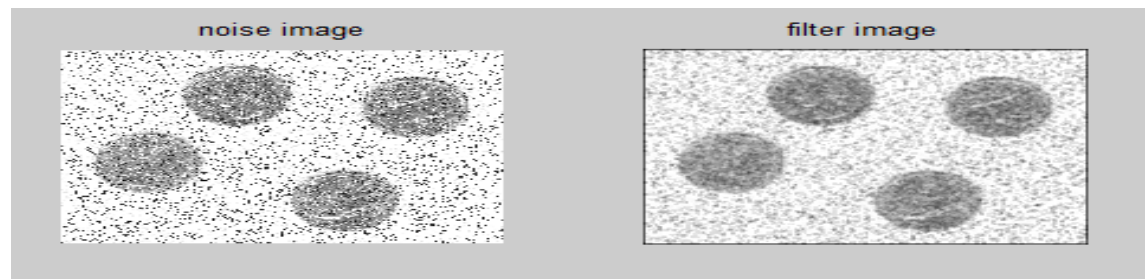

Fig. 1

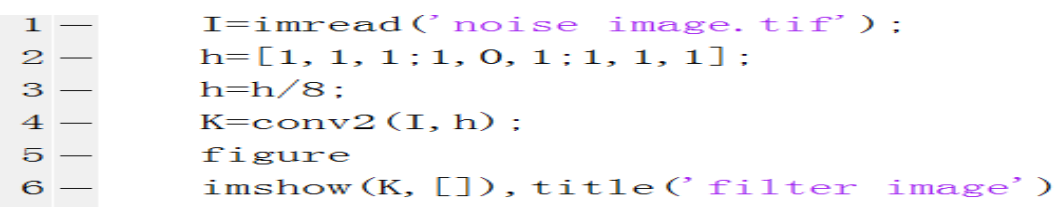

Fig. 2

Median filter. Median filter is also known as the minimum filter or the maximum filter, whose basic principle is to exchange the value of a point in digital images and digital sequence with the median value of each point in the neighborhood of the point. The original image of salt and pepper noise and Gaussian noise, and the results of image processing are shown in Fig.3. MATLAB code is shown in Fig. 4.

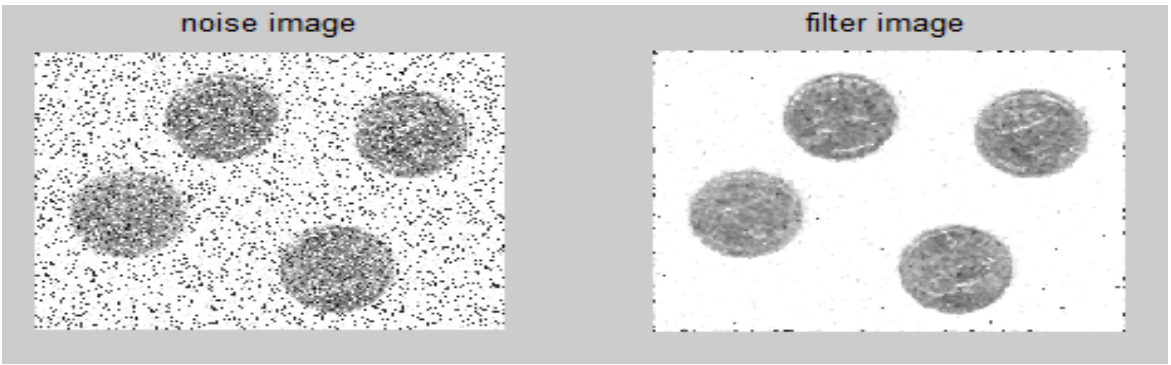

Fig. 3

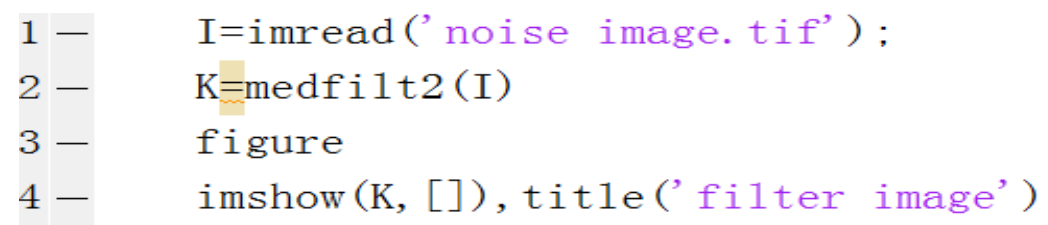

Fig. 4

\section{Fuzzy image processing}

Gaussian filter fuzzy processing. Gaussian filter is a process of weighted average to the whole image. The value of each pixel is obtained through itself and other pixel values in neighborhood after the weighted average. The specific operation of Gaussian filter is that a template or convolution/mask is used to scan each pixel in image and the weighted average gray value of the neighborhood, which is determined by a template, replaces the value of pixel in the center of the template. The original image of motion blur and the result of image processing are shown as Fig. 5. MATLAB code is shown in Fig. 6.

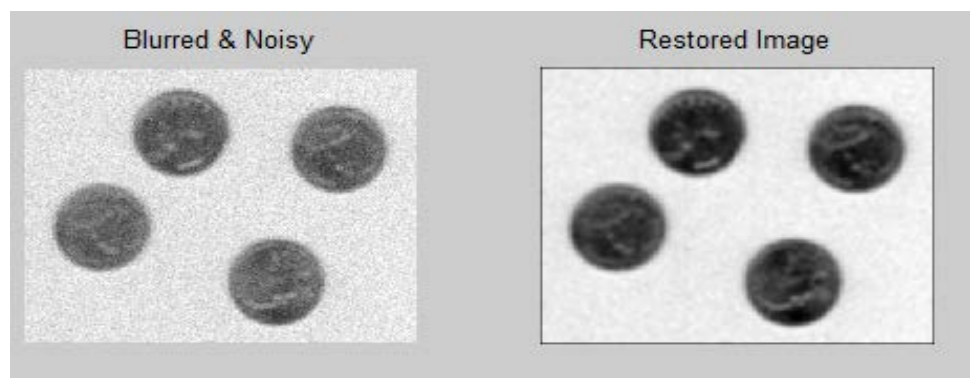

Fig. 5 


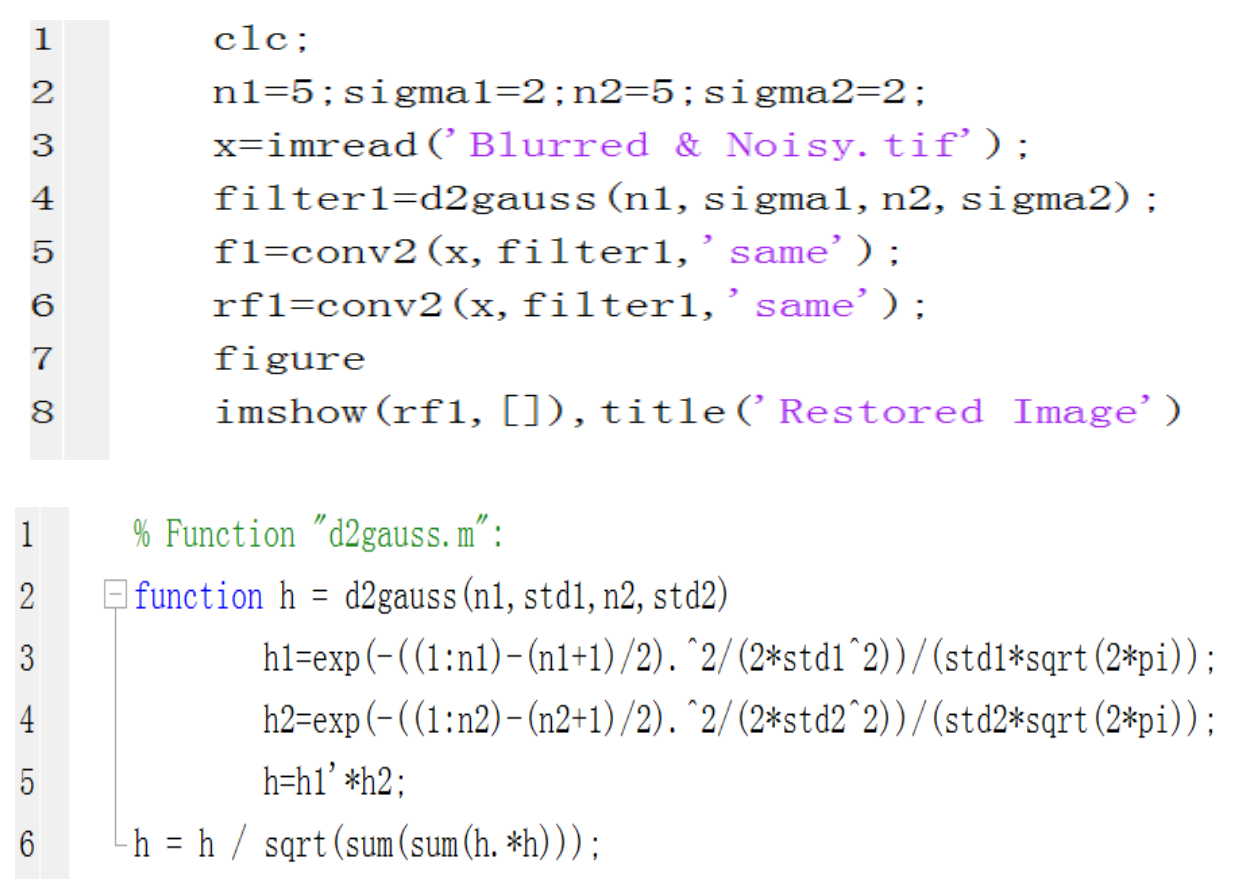

Fig. 6

Lucy-Richardson fuzzy processing. Lucy-Richardson algorithm belongs to iterative nonlinear restoration algorithm and the optimal estimation criterion is the maximum likelihood criterion that makes the greatest probability density. The nonlinear algorithm has an advantage compared with the linear class while suppressing noise amplification and retaining edge information of the image. The original image of motion blur and the result of image processing are shown as Fig. 7. MATLAB code is shown in Fig. 8.

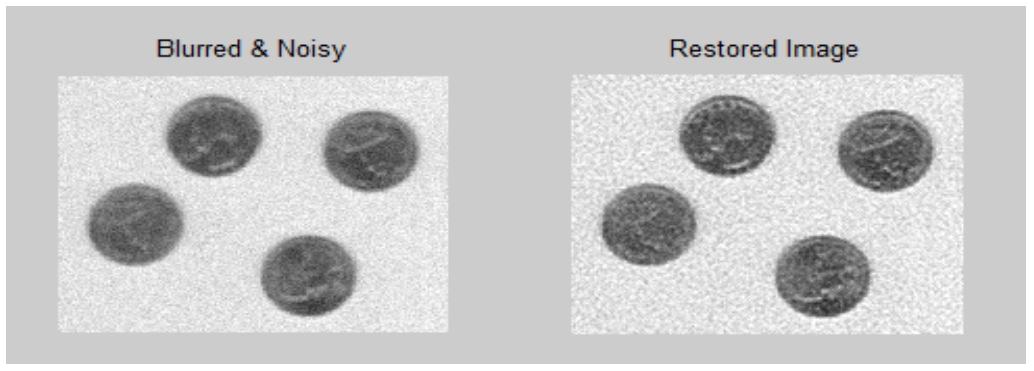

Fig. 7

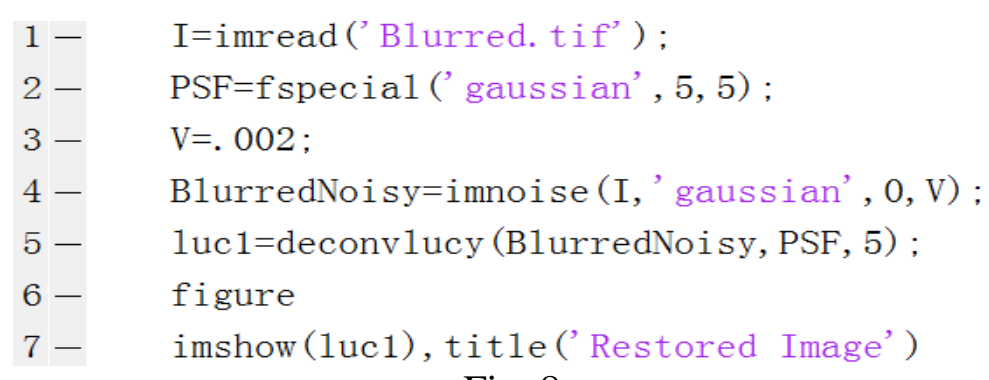

Fig. 8

\section{Conclusion}

In this paper, image preprocessing experiments are completely carried out with MATLAB software. There are various image preprocessing methods, which possess different advantages and disadvantages. According to this study, the conclusions can be made as follows:

Firstly, MATLAB function library is rich.

Secondly, the program is simple and easy, which is extremely effective for image preprocessing.

Thirdly, the workload is greatly reduced compared with the $\mathrm{C}++$ language program. 
However, some shortcomings are also found.

Firstly, the program has some limitations that the processing effect of pictures with complex elements is not ideal.

Secondly, a part of threshold should be adjusted based on the actual situation sometimes.

Thirdly, neighborhood average is better for the processing of Gaussian noise, but is not ideal for the salt and pepper noise treatment. Median filtering algorithm is better for salt and pepper noise processing, but is not ideal for the de-noising of Gaussian noise.

Fourthly, the complexity of Lucy-Richardson algorithm is high and there are problems of local convergence and algorithm stability.

\section{References}

[1] Hsiao Shih-Wen, Chuang Jiun-Chau. A reverse engineering based approach for product form design. Design Studies. Vol. 24 (2003), p. 155-171.

[2] Chau K W, Albermani F. A coupled knowledge-based expert system for design of liquid-retaining structures. Automation in Construction, Vol. 12 (2003), p. 589-602.

[3] Finnie Gavin, Sun Zhaohao. R5 model for case-based reasoning. Knowledge-Based Systems, Vol.16 (2003), p. 59-65.

[4] Yao Min, et al. Digital Image Processing, Machinery Industry Press (2006).

[5] Gonzalez. Digital Image Processing (MATLAB version). Beijing: Electronic Industry Press (2007).

[6] Rafael C. Gonzalez. Digital Image Processing. Beijing: Electronic Industry Press (2004).

[7] Chengbo Yu. Digital Image Processing and MATLAB Realization. Chongqing: Chongqing University Press (2003). 